\title{
ON A CLASS OF IMPLICIT DIFFERENTIAL INCLUSIONS
}

\author{
ZOUHUA DING \\ (Communicated by Dale Alspach)
}

\begin{abstract}
The existence of solutions is established for implicit differential inclusions $M\left(x^{\prime}\right)+C(x) \ni 0$ in $R^{n}$ involving the sum of a maximal monotone mapping $M$ and an upper semicontinuous mapping $C$ with compact, closed values. A question of Wenzel is answered in the affirmative.
\end{abstract}

\section{Introduction. Preliminaries}

Consider the initial value problem

$$
M\left(x^{\prime}\right)+C(x) \ni 0 \text { a.e., } x(0)=x_{0},
$$

where $M$ and $C$ are multi-valued mappings in $R^{n}$. Denote the solution set of (1) by $S(M, C)$. If $M$ is maximal monotone and $C$ is of subdifferential type, Wenzel proved in [1] that (1) has local solutions and noted: "it is not known to the author whether the conditions concerning $C$ may be weakened or not. Of course, there are trivial counter-examples if $C$ is not convex-valued. On the other hand, no counter-example seems to be known even in the case $C$ is allowed to be an upper semicontinuous (usc) convex-valued map." In this note we show the existence of solutions of (1) in case $C$ is an usc map with compact, convex values. We denote by $B_{r}\left(x_{0}\right)$ the open ball of $R^{n}$ with center at $x_{0}$ and radius $r>0$.

For the sake of convenience, we list the following lemmas (cf. [1], [3]).

Lemma 1 [3, Proposition 1]. Let $C: \overline{B_{r}\left(x_{0}\right)} \rightarrow 2^{R^{n}}$ be usc with compact, convex values. If $C$ is bounded, then there exists a sequence of usc functions $C_{n}: \overline{B_{r}\left(x_{0}\right)} \rightarrow$ $2^{R^{n}}$ which have compact and convex values and are such that:

1. $C(x) \subset C_{n}(x)$ for $n=1,2, \ldots$,

2. $C_{n+1} \subset C_{n}(x)$ for $n=1,2, \ldots$,

3. $\cap C_{n}(x)=C(x)$,

4. $C_{n}(x)=\sum_{i=1}^{p(n)} \lambda_{i}^{n}(x) C_{i}^{(n)}$, where $C_{1}^{(n)}, \ldots, C_{p(n)}^{(n)}$ are nonempty, compact, convex subsets of $R^{n}$, while the functions $\lambda_{i}^{(n)}$ form a Lipschitz continuous partition of unity of $\overline{B_{r}(0)}$.

Received by the editors September 1, 1992 and, in revised form, March 1, 1994.

1991 Mathematics Subject Classification. Primary 34A60; Secondary 34A09.

Key words and phrases. Maximal monotone operator, upper semicontinuous function, implicit differential inclusion. 
Lemma 2 [1, Theorem 3.1]. Let $M: R^{n} \rightarrow 2^{R^{n}}$ be maximal monotone and $C$ : $D \rightarrow R^{n}$ single-valued and continuous on an open set $D \subset R^{n}$. Assume, further, that $-C(D) \subset \operatorname{int} R(M)$. Then, for every $x_{0} \in D$, (1) has a Lipschitz continuous solution defined on an interval $[-\alpha, \alpha]$, for some $\alpha>0$.

The Lipschitz constant and the interval of definition of $x$ depend only on $x_{0}$. This is due to the following. Since if $M$ is maximal so is $M^{-1}$, it follows that $M^{-1}$ is convex-valued on $D\left(M^{-1}\right)$ and usc on $\operatorname{int} R(M)$ (cf. [1, Proposition 2.2]). Thus, $M^{-1}(-C(x))$ is usc and convex-valued on $D$. It follows that every local solution $x(t)$ of

$$
x^{\prime}(t) \in M^{-1}(-C(x)) \text { a.e, } x(0)=x_{0},
$$

can be extended at least to $[-d / L, d / L]$, where $L=\sup \left\{\|y\|: y \in M^{-1}(-C(D))\right\}$ and $d=\operatorname{dist}\left(x_{0}, \partial D\right)$, so that it is Lipschitz continuous there with $\left\|x^{\prime}\right\|_{\infty} \leq L$ (cf. [1, Remark on Proposition 2.1], or [2, Corollary 1 on page 129]).

\section{Main Result}

Theorem 1. Assume that $M: R^{n} \rightarrow 2^{R^{n}}$ is maximal monotone. Let $C: \overline{B_{r}\left(x_{0}\right)} \rightarrow$ $R^{n}$ be usc, with compact, convex values, and such that: $\|C(x)\| \leq K$ for every $x \in \overline{B_{r}\left(x_{0}\right)}$. Then there exists $\alpha>0$ such that (1) has a Lipschitz continuous solution on $[-\alpha, \alpha]$. Furthermore, the solution set $S(M, C)$ of $(1)$ is compact.

Remark. $\overline{B_{r}\left(x_{0}\right)}$ above may be replaced by an open set containing $x_{0}$.

Proof of Theorem 1. Let $C_{n}$ be the approximation of $C$ as in Lemma 1, and consider the inclusion

$$
0 \in M\left(x^{\prime}\right)+C_{n}(x), x(0)=x_{0} .
$$

Denote the solution set of this inclusion by $S\left(M, C_{n}\right)$ and the solution set of (1) by $S(M, C)$. If we assume that the sets $S\left(M, C_{n}\right)$ and $S(M, C)$ are nonempty and all the solutions in these sets are defined on the same closed finite interval $I \subset R^{n}$, then

$$
\bigcap_{n} S\left(M, C_{n}\right)=S(M, C)
$$

However, this relation can also be interpreted as follows: (a) if the left-hand side of (3) is nonempty and each $S\left(M, C_{n}\right)$ consists of solutions defined on $I$, then the lefthand side of (3) is contained in $S(M, C)$; (b) if the right hand side of (3) consists of solutions defined on I, then the right-hand side of (3) is contained in the left-hand side. In fact, (b) follows from the fact that $S(M, C) \subset S\left(M, C_{n+1}\right) \subset S\left(M, C_{n}\right)$. To show (a), let $x \in \bigcap_{n} S\left(M, C_{n}\right)$. Then

$$
0 \in M\left(x^{\prime}\right)+C_{n}(x) \text { for all } n,
$$

which is equivalent to

$$
x^{\prime} \in M^{-1}\left(-C_{n}(x)\right)
$$

for all $n$. Thus,

$$
x^{\prime} \in \bigcap_{n=1}^{\infty} M^{-1}\left(-C_{n}(x)\right) \subset M^{-1} \bigcap_{n=1}^{\infty}\left(-C_{n}(x)\right)=M^{-1}(-C(x)) .
$$


Hence $x^{\prime} \in M^{-1}(-C(x))$, or $0 \in M\left(x^{\prime}\right)+C(x)$. It follows that $x \in S(M, C)$, which proves (3). Now, choose $y_{i}^{n} \in C_{i}^{n}$ and consider the associated single-valued continuous function

$$
f_{n}(x) \equiv \sum_{i=1}^{p(n)} \lambda_{i}^{n}(x) y_{i}^{n} \in C_{n}(x)
$$

and the differential inclusion

$$
0 \in M\left(x^{\prime}\right)+f_{n}(x), x(0)=x_{0} .
$$

Denoting the solution set of (4) by $S\left(M, f_{n}\right)$, we observe that

$$
S\left(M, f_{n}\right) \subset S\left(M, C_{n}\right)
$$

This proves (a).

From Lemma 2, we know that (4) has a Lipschitz continuous solution defined on some interval $\left[-\alpha_{n}, \alpha_{n}\right], \alpha_{n}>0$, for each $n$, which can be extended to $\left[-d / L_{n}, d / L_{n}\right]$ so that it is Lipschitz continuous there with Lipschitz constant $L_{n}$. Here, for $B \equiv$ $B_{r}\left(x_{0}\right)$,

$$
d=\operatorname{dist}\left(x_{0}, \partial B\right)=r, L_{n}=\sup \left\{\|y\|: y \in M^{-1}\left(-f_{n}(B)\right)\right\}
$$

Since

$$
C(B) \subset C_{n}(B) \subset C_{1}(B), n=1,2, \ldots,
$$

and $C$ is usc, $\bar{B}$ is compact and $C_{1}(\bar{B})$ is compact, there exists an open ball $B_{\varepsilon}(0)$ such that

$$
C(B)+B_{\varepsilon}(0) \supset C_{1}(\bar{B}) \supset C_{2}(\bar{B}) \supset \cdots .
$$

Hence, we have an estimate for $L_{n}$ as follows:

$$
L_{n} \leq \sup \left\{\|y\|: y \in M^{-1}\left(-C(\bar{B})+B_{\varepsilon}(0)\right)\right\} \equiv L_{0} .
$$

Thus,

$$
d / L_{n} \geq d / L_{0}
$$

We conclude that we now have a common interval $\left[-d / L_{0}, d / L_{0}\right]$ and a common Lipschitz constant $L_{0}$ for all $n$. It is easy to see, by using the Arzelà-Ascoli theorem, that $S\left(M, C_{n}\right)$ is relatively compact in $C\left[-d / L_{0}, d / L_{0}\right]$. We are going to show that the set $S\left(M, C_{n}\right)$ is closed, and thus compact. Set

$$
I=\left[-d / L_{0}, d / L_{0}\right]
$$

and

$$
K \equiv\left\{x \in C(I): x \text { is Lipschitzian with constant } L_{0} \text { and } x(0)=x_{0}\right\}
$$

We define a set-valued mapping $F_{n}$ on $K$ as follows:

$$
F_{n}(x)=\left\{z \in K: z^{\prime} \in M^{-1}\left(-f^{(n)}(x)\right) \text { a.e. in } I \text {, for some } f^{(n)} \in C_{n}\right\} \text {. }
$$


We show that the graph of $F_{n}$ is closed in $C(I) \times C(I)$, where $C(I)$ is associated with the sup-norm. We do this for future reference and because the closedness of $S\left(M, C_{n}\right)$ will follow from a very similar proof. Unless otherwise stated, the convergence properties below are with respect to the sup-norm. Let $x_{k}^{(n)} \in K, z_{k}^{(n)} \in$ $F_{n}\left(x_{k}^{(n)}\right)$ be such that $z_{k}^{(n) \prime} \in M^{-1}\left(-f_{k}^{(n)}\right)$, and assume that $x_{k}^{(n)} \rightarrow \bar{x}^{n}, z_{k}^{(n)} \rightarrow \bar{z}^{n}$ as $k \rightarrow \infty$. Since the functions $f_{k}^{(n)}$ are Lipschitz continuous and $x_{k}^{(n)}$ are Lipschitzian solutions of (2), we have that $y_{k}^{(n)}=f_{k}^{(n)}\left(x_{k}^{(n)}\right)$ are also Lipschitz continuous. Thus, $y_{k}^{(n)}$ is relatively compact by the Arzelà-Ascoli theorem. It follows that a subsequence of $\left\{y_{k}^{(n)}\right\}$ (denoted again by $\left\{y_{k}^{(n)}\right\}$ ) converges to (some) $\bar{y}$. Letting $h_{k}^{(n)} \equiv z_{k}^{(n) \prime}(t) / L_{0}$, we see that the functions $h_{k}^{(n)}$ belong to the unit ball of $L^{\infty}\left(I, R^{n}\right)$, which is weakly* compact by Alaoglu's theorem because $L^{\infty}\left(I, R^{n}\right)$ is the dual of $L^{1}\left(I, R^{n}\right)$. Therefore, a subsequence of $h_{k}^{(n)}$ (denoted again by $h_{k}^{(n)}$ ) converges weakly* to some function $\bar{h}$ in $L^{\infty}\left(I, R^{n}\right)$. However, the operator of multiplication by the continuous function $c(t) \equiv L_{0}$ is continuous from $L^{\infty}\left(I, R^{n}\right)$ (associated with the weak* topology $\sigma\left(L^{\infty}, L^{1}\right)$ ) to $L^{1}\left(I, R^{n}\right)$ (associated with the weak topology $\sigma\left(L^{1}, L^{\infty}\right)$ ). For this fact we also refer to the proof of $[2, \mathrm{p} .14$, Theorem 4]. Thus, the sequence of functions $\left\{L_{0} h_{k}^{(n)}\right\}$ converges weakly in $L^{1}\left(I, R^{n}\right)$ to the function $L_{0} \bar{h}$. This implies that $h_{k}^{(n)}$ converges weakly in $L^{1}\left(I, R^{n}\right)$ to $\bar{h}$. Since

$$
z_{k}^{(n)}(t)-z_{k}^{(n)}\left(t_{0}\right)=\int_{t_{0}}^{t} h_{k}^{(n)}(s) d s
$$

we deduce that

$$
\bar{z}(t)-\bar{z}\left(t_{0}\right)=\int_{t_{0}}^{t} \bar{h}(s) d s
$$

Hence, for almost all $t \in I, \bar{z}^{\prime}(t)=\bar{h}(t)$. We observe now that the following properties hold.

1. $y_{k}^{(n)} \rightarrow \bar{y}$ a.e. $I$;

2. $h_{k}^{(n)} \rightarrow \bar{h}$ weakly in $L^{1}\left(I, R^{n}\right)$;

3. $M^{-1}$ is maximal monotone with convex and closed values.

Consequently, from the convergence theorem [2, page 60], we obtain that

$$
\bar{h} \in M^{-1}(-\bar{y}) .
$$

Note that since $y_{k}^{(n)}$ converges, $h_{k}^{(n)}$ converges weakly and $M^{-1}$ is usc with convex, closed values, we can get the same result by using Mazur's theorem. Thus, we obtain

$$
\bar{z}^{\prime} \in M^{-1}(-\bar{y}) \subset M^{-1}\left(-C_{n}(\bar{x})\right) .
$$

The last inclusion is derived from the following fact. Since $C$ is usc with compact and convex values, so that its graph is closed, we have that $\bar{y} \in C_{n}(\bar{x})$. We have proved that the graph of $F_{n}$ is closed. By using $x_{k}^{(n)} \in K$ instead of $z_{k}^{(n)} \in K$ above, we see that the set $S\left(M, C_{n}\right)$ is also closed. Since

$$
S(M, C)=\bigcap_{n=1}^{\infty} S\left(M, C_{n}\right)
$$


and $S\left(M, C_{n}\right) \supset S\left(M, C_{n+1}\right), n=1,2, \ldots$, we have that $S(M, C)$ is nonempty and compact. This tells us that the solutions of (1) exist and have the same interval of definition and the same Lipschitz constant. The proof is complete.

It is important to note here that it is possible to assume that the usc function $C$ has just convex, closed values if its domain is all of $R^{n}$. In fact, if this is the case, then we may use instead of Lemma 1 the construction of $[2$, p. 86 , Theorem 1]. That construction gives a structure similar to that of Lemma 1, based on the fact that such a mapping $C$ is $\sigma$-selectionable.

It would be also interesting to see extensions of Theorem 1 to time-dependent problems of the type

$$
M\left(x^{\prime}\right)+C(t, x) \ni 0
$$

in finite and infinite dimensional spaces. For example, it would be nice to see some results in this direction with the operator $M$ being $m$-accretive from a Banach space into itself.

The author wishes to thank the referee for his remarks and Professor A. G. Kartsatos, University of South Florida, for his helpful suggestions.

\section{REFERENCES}

1. G. Wenzel, On a class of implicit differential inclusions, J. Diff. Eq. 63 (1986), 162-182. MR 87i:34015

2. J. P. Aubin and A. Cellina, Differential Inclusions, Springer-Verlag, 1984. MR 85j:49010

3. C. J. Himmelberg and F. S. Van Vleck, A note on the solution sets of differential inclusions, Rocky Mountain J. of Math. 12 (1982), 621-625. MR 84b:34019

Department of Mathematics , University of South Florida, Tampa, Florida 33620 5700

E-mail address: ding@chuma.usf.edu 